\title{
Stratégies discursives de persuasion dans la critique de cinéma comme activité rhétorique
}

\author{
Discursive strategies of persuasion in a film review \\ as a rhetorical activity
}

\author{
Dominika Topa-Bryniarska \\ Université de Silésie \\ dominika.topa@us.edu.pl
}

\begin{abstract}
The present paper deals with the discursive construction of persuasiveness in media language. Based on eighty reviews of two French comedies Intouchables (2011) and Qu'est-ce qu'on a fait au Bon Dieu? (2014), different components of the genre 'film review' - relatively little explored until recently - are established in the light of two operations of metonymy exploring the rhetorical argument from community. As the operations mentioned contribute to influencing the addressees' will and decisions, they perform the phatic function linked to the rhetoric principle of movere and delectare. Accordingly, it is important to notice that the persuasive strategies reflect some methods used in advertising discourse, especially with regard to indirect means of interpretation, values and emotions, which become starting points for the deliberative dimension of the film review. These parameters permit to reduce the information verification process by applying colloquial expressions, directive speech acts, collocative pronouns and generic quantifiers, since they stem from the trustworthiness relation connected with the peripheral route of persuasive influence.
\end{abstract}

Keywords: discursive strategies of persuasion, film review, phatic function, metonymy, deliberative dimension of the film review, trustworthiness relation.

\section{INTRODUCTION}

Dans ce travail, nous nous proposons d'étudier les stratégies discursives de persuasion dans la critique de cinéma perçue comme activité socio-discursive de nature pragmatique et institutionnelle que nous classifions parmi les genres institués de 
type routinier. Les paramètres de ces genres relèvent « de la stabilisation, sous forme de normes, de contraintes liées à une activité verbale qui s'exerce dans une situation sociale déterminée " (Maingueneau, 2007, para. 7). Une telle dimension permet d'instaurer le cadre d'un contrat de communication médiatique qui impose aux locuteurs un certain nombre de conditions modalisant l'enjeu de l'échange communicatif, soit les stratégies utilisées, l'identité des partenaires et leurs domaines du savoir (Charaudeau, 2005).

Le corpus qui servira à notre démonstration regroupe quatre-vingts critiques identifiées sur divers sites Internet consacrés au cinéma et sur ceux de journaux français et francophones. La première partie du corpus se compose de textes publiés entre septembre 2011 et décembre 2012 qui se réfèrent à la fameuse comédie française de l'année 2011 - Intouchables ; l'autre partie du corpus présente la comédie Qu'est-ce qu'on a fait au Bon Dieu ? (2014) et regroupe les critiques publiées entre mars 2014 et mars $2015^{1}$. À partir des textes indiqués, nous ne pouvons évidemment pas prétendre à l'exhaustivité de notre travail. C'est pourquoi notre analyse n'est que préliminaire et - vu l'étendue des problèmes évoqués - nécessite une continuation des recherches.

La complexité des phénomènes discursifs, qui s'articulent à la critique de cinéma - genre peu relativement exploré même s'il représente l'univers dynamique des médias contemporains orientés vers l'efficacité communicationnelle et l'hétérogénéité formelle - prête à se poser, à notre avis, des questions concernant les mécanismes persuasifs formant notre perception : l'émetteur doit miser sur les goûts, les croyances, les connaissances de ceux qu'il veut persuader pour élaborer une stratégie qui puisse les atteindre. C'est là que réside le potentiel de création et/ou de renforcement de diverses attitudes, opinions collectives et représentations afin de produire chez le destinataire, d'après Amossy (2008), un changement d'opinion, aussi bien affectif que cognitif, par rapport à un point de vue donné ${ }^{2}$. Nous considérons ainsi la notion de stratégies discursives de persuasion (SDP) en tant qu'activité argumentative, qui, tout en dépassant un simple acte de langage, reste

\footnotetext{
${ }^{1} \mathrm{Vu}$ la dimension du corpus, un échantillon de trente-cinq textes a été retenu dont nous énumérons les sources : Le Point, Le Monde, Télérama, La Croix, La Presse, France Soir, Le Soleil, Le Figaro, Moustique, Le Journal du Dimanche, Marianne, Le Suricate, Paris Match, Les InRocKs, Journal La Marne, Le Grand Soir, Abusdecine.com, Cinematon.com, Oblikon.com, Filmosaure.com, Cinemovies.fr, LaCritiquerie.com, Filmosphere.com, Mondociné.net, Cinema.jeuxactu.com, Cineeurope.org, La Vie.fr, Zamanfrance.fr, Avoir-Alire.com, WatzUp.fr, MceTv.fr, CinéBuzz.fr, Cahiers Libres. fr, CineSeriesMag.fr. Les dates de parution des critiques étudiées (là où c'est possible) sont aussi signalées au fur et à mesure de leur analyse.

${ }^{2}$ Nous n'allons pas aborder ici le problème de la fameuse distinction entre convaincre et persuader qui n'est pas utile à notre approche. À titre d'exemple, nous pouvons citer l'ouvrage de Breton (2008) qui accentue la dissociation de l'acte de convaincre et de l'acte d'informer (sur la discussion autour des positions variées dans le cadre de l'étude argumentative, $c f$. Micheli, 2012).
} 
plus restreinte que la notion de compétence linguistique au sens chomskien (cf. aussi Lugrin, 2006).

Nous croyons en outre que les sens véhiculés par les SDP contribuent à (re)construire la réalité sociale à travers des procédures servant à capter l'attention du plus grand nombre possible de récepteurs (enjeu de captation, Charaudeau, 2005). Nous nous référons ici au sens large de l'acte de persuader issu de la rhétorique classique et inscrit dans la lignée perelmanienne, où la notion d'analyse de discours - comme le notent Charaudeau et Maingueneau (2002) - correspond à l'adresse rhétorique (ang. public address) plutôt qu'à la définition foucaultienne (cf. Doury \& Plantin, 2015). Nous nous appuyons aussi sur l'optique argumentative proposée par Amossy $(2008,2012)$ dans la mesure où nous nous intéressons avant tout aux discours à visée argumentative dont l'objet principal est effectivement de persuader l'autre. Nous empruntons également la notion d'éclairage à l'approche argumentative de Grize (2004), car le choix du lexique ne sert pas seulement à présenter le point de vue du locuteur, mais aussi à mettre en oeuvre l'orientation pragmatique du discours.

Ainsi, en nous situant aussi bien dans le cadre de la linguistique fonctionnelle du discours médiatique ( $c f$. Stöckl, 2015) qu' « au carrefour de l'argumentation rhétorique et de l'analyse du discours » (Amossy, 2015), nous nous proposons de dégager des unités linguistiques mettant en discours le fonctionnement argumentatif du pathos et de l'ethos dans la critique de cinéma qui, comme les genres d'opinion, réalise en particulier deux types de visées : factitive et persuasive ( $c f$. Brin, Charron, \& Bonville, 2004). Ces deux visées forment ensemble la visée délibérative (VD) dont nous parlerons plus tard. Notre travail n'a donc d'autre ambition que d'investiguer les paramètres rhétoriques de movere et delectare présidant à l'entreprise de persuasion ancrée dans la VD qui, sous forme de ses stratégies, est proche de ce que l'on peut observer dans le discours publicitaire visant à instituer le public en cible et destinataire du discours. À cet effet, nous essaierons d'abord de passer en revue les spécificités générales de la critique de cinéma inscrites dans la dimension délibérative du genre. Ensuite, à l'appui de l'étude de cas qualitative, nous montrerons comment ce dispositif délibératif agit discursivement pour établir une connivence socioculturelle avec les récepteurs.

\section{VISÉE DÉLIBÉRATIVE DE LA CRITIQUE À TRAVERS LES STRATÉGIES DISCURSIVES DE PERSUASION}

Étant donné que le genre de discours est déterminé aussi bien par la situation de communication que par la finalité du contrat de communication médiatique, la critique de cinéma - comme toute autre critique culturelle (Rieffel, 2006) - a pour objet de fournir une évaluation motivée du film pour tous ceux qui envisagent peut- 
être de le voir (Baud, 2003). La critique s'apparente ainsi à un type particulier de publicité (ou d'anti-publicité) du film, comprenant dans sa structure des expressions valorisantes.

D'un point de vue pragmatique, la critique de cinéma doit donc servir le public, car le journaliste y explique les valeurs du film (jeu d'acteur, cadrage, montage, musique, photo, éclairage, etc.) en encourageant ou en dissuadant le récepteur d'aller au cinéma. D'un point de vue rhétorique, la réalisation du but relevant du contrat de communication se trouve toujours en tension entre deux types d'enjeux distingués par Charaudeau (2005) : enjeu de crédibilité dont le rôle est de faire savoir (visée d'information) et enjeu de captation visant à faire croire (visée incitative) et faire ressentir (visée pathémique) pour attirer l'attention du récepteur. Nous y avons donc affaire à une rhétorique interprétative où l'évaluation est précédée d'une analyse critique effectuée par l'émetteur, ce qui se concrétise dans trois composantes structurelles du genre : partie informative, partie analytique et partie évaluative (Pietrzak, 2014 ; Wolny-Zmorzyński, Kaliszewski, Snopek \& Furman, 2014).

Comme la critique de cinéma constitue une sorte de compromis entre la volonté de l'émetteur de dire tout ce qu'il croit nécessaire au sujet du film et son souhait d'être lu, elle s'affiche - à l'instar du discours publicitaire contemporain - tout autant comme un espace de circulation de significations et de valeurs que comme un « prescripteur » de biens de consommation, c'est-à-dire les films à voir ou à éviter. Cette singularité de la critique s'explique majoritairement par sa double finalité : capter l'attention (visée persuasive) et inciter à faire (visée factitive). Pour parvenir à ces fins, l'émetteur déploie donc les SDP dont la fonction - à l'instar du discours publicitaire - est de provoquer un effet d'attraction et de captation en mobilisant différents procédés narrativo-descriptifs de connivence qui vont s'étayer sur des entités variées telles que croyances, valeurs, représentations, etc. et qui vont miser sur la relation de fiabilité et l'empathie pour construire une communauté discursive d'appartenance. Puisque ces stratégies sont marquées par les effets que le discours peut produire, elles constituent le reflet du cadre de pensée de la communauté mentionnée. D'un tel point de vue, les SDP font partie de la composante rhétoricopragmatique de la critique de cinéma qui veut se faire remarquer en oscillant entre originalité et accessibilité, ce qui donne lieu à l'activation d'autres fonctions : expressives, phatiques et cognitives, soumises à la catégorie supérieure du dispositif délibératif. Ce dernier se compose de l'acte illocutoire primaire de type directif ( $\mathrm{Je}$ vous conseille/déconseille de voir le film $X$ ) auquel sont soumis des actes illocutoires secondaires de type constatatif - louangeurs ou dépréciatifs - qui correspondent à la dimension démonstrative (épidictique) du genre.

Le genre de la critique fonctionnerait ainsi en tant que catégorie communicationnelle qui, sur le plan discursif, prend la forme de diverses stratégies persuasives centrées sur l'intention de l'émetteur. En d'autres termes, l'émetteur est conscient du fait - ce qui découle d'ailleurs du contrat même de communication médiatique - que 
les récepteurs-spectateurs traiteront sa critique comme un outil à fin délibérative où l'on incite à faire, en informant sur une oeuvre artistique (partie informative de la critique) et en expliquant si celle-ci est digne d'intérêt (partie analytique et évaluative). Une telle perspective permet de traiter la critique de cinéma comme macroacte performatif à visée délibérative qui enferme le potentiel persuasif et actionnel du genre, soit son visée persuasive et factitive. C'est pourquoi le dispositif délibératif de la critique englobe aussi des éléments démonstratifs (épidictiques) ${ }^{3}$, présentant les valeurs esthétiques du film, où l'émetteur peut faire preuve de son expertise lorsqu'il loue ou blâme les différents éléments du film. La VD de la critique de cinéma sert donc à atteindre un but perlocutoire précis, soit la décision du public d'aller voir le film ou bien de ne pas le faire.

Comme nous voulons décrire les effets visés (possibles) d'une mise en discours similaire aux techniques du discours publicitaire, notre intérêt portera principalement sur l'enjeu de captation qui - à notre sens - prime dans la critique de cinéma d'aujourd'hui. Cela est lié à la spécificité des médias contemporains orientés vers l'infotainment, le principe phatique de séduire et la délibération avec le public, à l'instar des techniques utilisées dans l'argumentation publicitaire ( $c f$. Bonhomme $\&$ Pahud, 2013). De ce fait, en voulant négocier du sens et jouer les interactions avec les récepteurs, la critique influence les goûts du public par le biais des stratégies qui opèrent sur le dialogisme, la subjectivité, la polémique, l'expressivité et la singularité pour fournir du divertissement, de l'émotion, de la valorisation ou de l'esthétique ( $c f$. Stöckl, 2015). La VD de la critique activera ainsi la paire de faire croire et de faire ressentir due à l'activation d'associations culturelles, des présupposés, des sous-entendus, basés principalement sur le jeu entre l'ethos et le pathos. Ainsi le potentiel persuasif de la VD ne se réduira pas en seuls mots ou phrases mais à tout un assemblage d'hypothèses et d'inférences qui s'ajoutent à ce qui est dit et dont les phrases et les mots sont «déclencheurs» ( $c f$. aussi Jacobs, 2015). En conséquence, afin «d'orienter la volonté et de déterminer l'action » (Plantin, 2011, p. 18), l'émetteur peut, à certain degré, contrôler « la psychologie » de la réception du message en choisissant des SDP variées qui contribuent à segmenter la réalité décrite en évoquant les communautés discursives qui appartiennent à cette "programmation » du réel. Puisque le sujet est large, nous nous pencherons sur un type de stratégies, à savoir celles qui convoquent l'argument de communauté grâce auquel on peut renforcer ou affaiblir l'opinion du récepteur à l'égard de l'objet présenté tout en établissant une relation de fiabilité due à la mise en avant de la fonction phatique dans l'acte de persuader.

\footnotetext{
${ }^{3}$ Nous ne nous intéressons pas ici aux critiques de cinéma à visée démonstrative écrites par des spécialistes pour des spécialistes. Le but du discours démonstratif est de vanter ou de blâmer l'objet de discours (en termes d'esthétique) par la consolidation des valeurs que le public devrait accepter (cf. Graff \&Winn, 2006).
} 


\section{3. ÉTUDE DE CAS : LES STRATÉGIES EXPLOITANT L'ARGUMENT DE COMMUNAUTÉ}

Les stratégies indiquées contribuent à construire et à reconstruire le réel à travers l'enjeu de captation inscrite dans la VD de la critique de cinéma. Dans cet enjeu, la visée factitive de la mise en scène de l'information se traduit par la visée persuasive : pour entreprendre une action, le lecteur doit se sentir intéressé par le contenu du message sans pourtant se rendre compte que ce sont les médias qui y conduisent. C'est aussi l'une des «facettes » de l'acte de persuader où l'on suggère un choix : il faut faire en sorte que le récepteur se sente constamment engagé dans la communication et qu'il ait l'impression de se persuader lui-même. Pour ce faire, l'émetteur recourt à la mise en relief ou bien au masquage de certaines données, donc en soustendons ce que nous pouvons appeler architecture de l'information et ce que Grize (2004) entend par notion d'éclairage : « les objets de discours doivent être éclairés, ce qui revient à mettre en évidence quelques-unes de leurs facettes et à en occulter d'autres et tout éclairage colore ce qu'il illumine, ce qui découle du fait qu'il se sert des préconstruits culturels qui ne sont jamais neutres » (p. 42).

Ce procédé correspond en l'occurrence au choix des termes dont le but est de présenter le film en tant qu'objet agréable ou désagréable, utile ou inutile selon qu'on veut le critiquer ou vanter. On cherche donc à faire croire en essayant de donner une portée maximale, voire universelle, à l'objet évalué ; on tend aussi à faire ressentir en mobilisant un effet de groupe fondé sur l'identification à des valeurs similaires. Un tel procédé ressemble à ce dont se sert le discours publicitaire lorsqu'il accentue les performances et les spécificités des biens de consommation quant à leur quantité et qualité qui constituent des grands lieux ou super valeurs (topoï) de l'argumentation (Breton, 2008 ; Perelman, 2000 ; Robrieux, 2000). Selon Charaudeau (2005), la focalisation dramatisante du récit médiatique, par une opération de métonymie, réduit l'ensemble des composantes d'un événement à l'une de ses parties, abolissant momentanément le reste du monde. De plus, en tant que rapport de contiguité, la métonymie contribue - sur le plan rhétorique - à modeler la perception par l'établissement de faire croire et de faire ressentir, et dans ce sens, elle pourra être regardée non seulement comme un outil de persuasion, mais aussi comme celui de cognition (Kövecses, $2011)^{4}$. Il est intéressant de noter que l'argument de communauté, qui fait appel à « des présupposés communs »(Breton, 2008, p. 91), s'assimile à deux types de métonymies qui sont à l'origine de telles opérations persuasives que :

\subsection{GÉNÉRALISATION}

L'opération de généralisation recourt au lieu (topos) de la quantité en s'appuyant sur une métonymie qui agit selon le lieu commun suivant : si la majorité des gens

\footnotetext{
${ }^{4} C f$. aussi les arguments fondés sur la structure du réel (Perelman, 2000).
} 
pensent que telle ou telle chose est bonne/mauvaise, cette opinion a de fortes chances d'être vraie ou au moins probable. Une telle perspective permet « d'éclairer » l'objet décrit sous l'angle de l'argumentation ex consensu gentium qui peut être mise en discours par des quantificateurs génériques de type tous, chacun, des pronoms inclusifs on et nous (aussi sous forme interrogative), des adjectifs numéraux sous forme de grandes données statistiques ou grands chiffres :

(1) c'est maintenant connu de tous ; Le genre de type rigolard que tout le monde voudrait avoir comme ami. (Le Soleil, 11.04.2012)

(2) (...) une succession d'épisodes tape-à -l'oeil destinés à faire saliver le consommateur qui sommeille en chacun de nous. (Le Grand Soir, 6.12.2011)

(3) On y retrouve tous les ingrédients des grands succès de la comédie française (...) (Le Point, 1.11.2011)

(4) Oui, ce racisme que l'on pardonne à tous (...) (Oblikon.com, date non mentionnée, consulté le 17.12. 2016)

(5) Tenons-nous là le Rain Man français ? (Cinema.jeuxactu.com, 15.11.2011, consulté le 18.12.2016)

(6) Car si on invite toutes les représentations à la fête, ça change tout, non ? (Mondociné.net, date non mentionnée, consulté le 16.12.2016)

(7) Que demander de plus à une comédie qui parvient à nous faire travailler l'ensemble des zygomatiques toutes les trente secondes ? (LaCritiquerie.com, 9.04.2014, consulté le 21.12.2016)

(8) Illustration de notre mal-être ? Chaînon manquant à ce lien social qui fait défaut à tant d'entre nous? (Moustique, 27.12.2011)

(9) Le piquet est planté, nous avons affaire à une comédie française, bien de chez nous (...) (Journal La Marne, 16.04.2014)

(10) Comme nous sommes au cinéma, tout finit évidemment bien. (Le Monde, 4.05.2014)

(11) (...) chacun saura se reconnaître malgré lui dans tel cliché ou telle expression borderline (...) (Filmosaure.com, 8.05.2014, consulté le 19.12. 2016)

(12) (...) le film d'Eric Toledano et Olivier Nakache, a déjà dépassé la barre des cinq millions de spectateurs. (Marianne, 16.11.2011)

(13) (...) une belle performance avec plus de 4 millions d'entrées. (Cinematon.fr, 9.11.2011, consulté le 12.12.2014)

(14) Intouchables a déjà été vendu par Gaumont dans plus de 40 pays (...) (Cineeurope.org, 31.10.2011, consulté le 16.11.2016)

(15) Plus de 6,5 millions d'entrées en trois semaines. Qu'est ce qu'on a fait au Bon Dieu ? est promis à un bel avenir au box-office. (Paris Match, 12.05.2014)

(16) Dix acteurs, dix millions d'entrées. (Le Figaro, 13.06.2014)

(17) Dès le premier jour de sa sortie, Qu'est-ce qu'on a fait au bon dieu ? a fait plus fort qu'Intouchables avec plus de 200000 tickets vendus (...) (Télérama, 6.05.2014)

(18) Moins de trois mois après, c'est la barre des 10 millions de spectateurs conquis qui est atteinte. (Mondociné.net, date non mentionnée, consulté le 16.12.2016) 
Comme nous pouvons l'observer dans les exemples cités, les SDP basées sur l'argument de communauté ont pour but de maintenir l'intérêt des récepteurs en incitant chez eux les mêmes émotions et le même enthousiasme que le journaliste éprouve lui-même. Les récepteurs, concernés par le caractère inclusif des pronoms et des quantificateurs génériques, semblent plus facilement adhérer à l'opinion de l'émetteur qui rêvet une forme plus généralisée. Une telle perspective permet de consolider l'adhésion du public à des valeurs et des croyances déterminées non seulement pour faire croire et faire ressentir mais aussi pour assurer l'engagement du public dans la communication, ce qui est ensuite maintenu par la mise en discours de la relation de fiabilité que Petty et Cacioppo (1986) regroupent sous le nom de voie périphérique de traitement de l'information ${ }^{5}$.

L'emploi de la forme interrogative dans (5-8) contribue également à consolider l'engagement du public, car ces questions n'ont d'autre but que de persuader le récepteur : elles contiennent déjà un élément évaluatif qui déclenche la réponse implicite ancrée dans les connaissances que l'émetteur veut faire partager avec son public. Nous y voyons à quel point le démonstratif et le délibératif s'imbriquent l'un dans l'autre parce que l'acte de conseiller s'appuie sur celui d'évaluer et vice versa. C'est ainsi que se fonde une liaison entre l'émetteur et son public : on tend à conduire les lecteurs à se construire une opinion qui reposera sur un jugement de valeur bien concret, véhiculé par les termes employés. Ce jugement peut ensuite servir d'appui au déclenchement des émotions que l'émetteur cherche à projeter sur son public. Aussi est-il possible d'y remarquer une posture énonciative due à l'imputation (Brin et al., 2004) à l'aide de laquelle l'émetteur peut « se mettre à la place de l'autre, de lui prêter des intentions (fondées ou non), des états (réels ou pas), des valeurs (vérifiées ou supposées) ».

Remarquons que l'extension dénotative des pronoms inclusifs et des quantificateurs génériques ne peut être interprétée qu'à l'aide du contexte, d'où sa capacité à neutraliser la diversité des récepteurs par une objectivation du jugement. Ces formes donc - fonctionnant ici en tant qu'arguments plus convaincants qu'une seule voix du journaliste - permettent de crédibiliser la position de l'émetteur comme critique et membre d'une plus vaste communauté. Un tel procédé, encore renforcé par l'utilisation des adjectifs numéraux (données statistiques et grands chiffres), contribue à l'automatisation de la réception du message par le fait d' "éclairer " l'objet de discours à l'appui des structures s'adressant directement à l'imagination des récepteurs. Ces structures permettent de dynamiser la réalité pour la rendre plus facilement acceptable. On peut ainsi sous-entendre que le film évalué vaut les qua-

\footnotetext{
${ }^{5}$ On note ici l'activation de la voie périphérique d'argumentation persuasive dont parlent Petty et Cacioppo (1986) dans leur Modèle de Probabilité d'Elaboration (Elaboration likehood model, E.L.M.). La relation de fiabilité (trustworthiness) correspond à l'une des spécificités de la source d'argumentation, à côté de l'expertise (expertise) et de l'attractivité (attractiveness).
} 
lités qui lui sont assignées : sous forme de raisonnement enthymématique, il est donc possible d'inférer que si ce film a déjà dépassé la barre des cinq millions de spectateurs (12), et qu'il a été vendu dans plus de 40 pays (14), il doit être une bonne comédie pour tous. De ce fait, en justifiant que le film est une oeuvre magnifique, l'émetteur suggère que cette oeuvre est universelle. Il a recours de cette façon au lieu commun que ce qui est universel est bon, ce qui lui permet d'englober l'ensemble des spectateurs potentiels, à savoir une communauté socioculturelle concrète.

\subsection{INTENSIFICATION}

Dans l'opération d'intensification, l'émetteur puise dans le lieu (topos) de la qualité par le biais duquel il essaie d'imposer son système de valeurs en utilisant une métonymie intensifiante qui se sert de trois types de structures : expressions absolues (totalisantes) sous forme de superlatifs, syntagmes affectifs appartenant aussi bien au registre standard de la langue qu'au registre populaire (familier) et actes directifs :

(19) (...) faites servir le tout par des acteurs au meilleur de leur forme. (Cineuropa.org, 31.10.2011, consulté le 16.08.2015)

(20) C'est aussi le meilleur moyen d'engranger les entrées. (Paris Match, 12.05.2014)

(21) (...) il est tout simplement le meilleur comédien français actuellement, le comédien avec le plus gros capital sympathie. (Cinema.jeuxactu.com, consulté le 17.12.2016)

(22) (...) les dialogues les plus osés. (Abusdecine.com, 2.11.2014, consulté le 16.12.2016)

(23) Qu'est-ce qu'on a fait au Bon Dieu ? s'avère la comédie la plus populaire de l'Hexagone (...) (La Presse, 31.07.2014)

(24) Qu'est-ce qu'on a fait au Bon Dieu ? est une excellente comédie (...) (WatzUp.fr, 14.04.2014, consulté le 17.12.2016)

(25) Machine à rire implacable (...) (Avoir-Alire.com, 27.03.2015, consulté le 20.12.2016)

(26) une savoureuse brochette de jeunes comédiens (Mondociné.net, date non mentionnée, consulté le 16.12.2016)

(27) la séquence d'ouverture est une idée tout simplement géniale (...) (Filmosphere.com, date non mentionnée, consulté le 20.12.2016)

(28) subtilité de cet acteur magique (Le Figaro, 2.11.2011)

(29) le miracle de la famille (La Vie.fr, 13.05.2014, consulté le 18.12.2016)

(30) une comédie drôlissime réussie (MceTv.fr, date non mentionnée, consulté le 20.12.2016)

(31) les raisons d'un triomphe (Le Figaro, 13.06.2014)

(32) étrange binôme de « sales gosses » (La Croix, 1.11.2011)

(33) la nounou idéale (Les InRocKs, 1.11.2011)

(34) Ce grand échalas à la voix acidulée pète la forme (...) (Le Point, 1.11.2011)

(35) Pourquoi ça cartonne. (Les InRocKs, 21.04.2014)

(36) cette comédie franchouillarde qui a l'air bien sympatoche. (Cahiers Libres.fr, 3.06.2014, consulté le 21.12.2016)

(37) Christian Clavier fait l'affaire en vieux réac. (Le Suricate, 15.04.2014) 
(38) Si vous ne l'avez pas vue dans la bande-annonce ou si vos amis ne vous l'ont pas encore racontée, dépêchez-vous d'aller voir Intouchables (...) (France Soir, 4.11. 2011)

(39) Cette comédie va vous faire rire aux éclats et vous arracher des larmes, n'en doutez pas une seconde ! (Le Journal du Dimanche, 29.10.2011)

(40) Bref, oubliez vos propres préjugés envers la comédie franchouille et tentez le diable, il y a matière à passer un excellent moment de divertissement ludique (...) (Avoir-Alire.com, 27.03.2015, consulté le 20.12.2016)

(41) allez, ça vaut bien un article de plus ! (Cinemovies.fr, 23.12.2011, consulté le 17.12.2014)

(42) Ce qui en fait une comédie savoureuse et rafraîchissante, à consommer sans modération! (Zamanfrance.fr, 25.04.2014, consulté le 27.12.2016)

(43) Pas de temps mort, une salle hilare, bref : de quoi passer un bon moment. (CinéBuzz.fr, 15.04.2014, consulté le 21.12.2016)

(44) Qu'est-ce qu'on a fait au Bon Dieu ? est à ranger dans la case du bon divertissement. (CineSeriesMag. fr, date non mentionnée, consulté le 19.12.2016)

En décrivant les caractéristiques du film en termes de superlatifs et d'adjectifs subjectifs affectifs (Kerbrat-Orecchioni, 1980) comme magique, génial, triomphe, miracle, excellente, implacable, savoureuse, drôlissime, on persuade non seulement par une réaction émotionnelle de l'émetteur face à l'objet décrit mais aussi par des présuppositions valorisantes qui doivent suggérer au public l'ensemble d'une zone axiologique à forte tonalité délibérative : le film est décrit comme le meilleur de son genre, donc, vu ses qualités, il saura satisfaire les goûts des spectateurs les plus variés. Cela présuppose également que les comédies françaises tournées avant Intouchables étaient moins réussies. Le procédé d'évaluation (Brin et al., 2004) ainsi activée permet d'obtenir un effet de dynamisation et de coloration de la réalité pour produire plus d'impression sur le public. Une telle technique doit persuader le récepteur de la justesse de l'opinion prononcée (faire croire) renforcée par l'apport émotionnel que les termes utilisés véhiculent (faire ressentir). Les superlatifs et les syntagmes affectifs permettent alors d'introduire un jugement de valeur et de susciter certaines émotions qui servent à établir un point de référence commun ainsi qu'à établir la relation de fiabilité. Cette relation a principalement trait à la réduction de la distance entre l'émetteur et le récepteur, ce qui est particulièrement visible dans le recours à l'oralité et l'emploi des termes du registre familier ou populaire de la langue comme le montrent les exemples (32-37). C'est par cette adaptation culturelle et linguistique - très pragmatiquement efficace - que l'émetteur veut générer des sentiments de proximité en créant son ethos d'amitié afin de provoquer une attitude positive chez son public puisqu'on a tendance à croire que ceux qui parlent «notre » langue sont plus dignes de confiance.

En ce qui concerne l'usage des actes directifs - aussi bien directs où l'on s'adresse explicitement au public comme dans (38-40) qu'indirects dans (41-44) - 
ils ont pour but de mener le public à suivre le conseil de l'émetteur, ce qui correspond au concept de prescription (Brin et al., 2004) au moyen duquel le journaliste s'adresse aux lecteurs en leur demandant d'écouter ses avis ou ses recommandations. Les actes directifs, par leur caractère phatique et expressif, sont « plus chargés émotionnellement que les énoncés axiologiquement neutres » (Kerbrat-Orecchioni, 2016, p. 28), ce qui - avec tous les autres moyens d'intensification - aide à entrer le public dans l'univers de l'émetteur. De tels procédés s'apparentent à bien des égards aux techniques d'amalgame référentiel que nous observons aussi dans le discours publicitaire : on cherche à incorporer le produit dans les réactions et les actions de l'utilisateur potentiel pour orienter l'interprétation du discours vers faire ressentir et faire croire. Par conséquent, la mise en discours du dispositif délibératif a pour objet d'inviter le plus grand nombre de récepteurs possible à se solidariser avec l'expérience de spectateur décrit par le journaliste.

\section{POUR CONCLURE}

Comme notre perception du monde est relative, la (re)construction de la réalité dans le cadre de la communication de masse constitue une forme d'expression complexe réalisée à travers plusieurs stratégies discursives dont une partie, vu l'ampleur de ce travail, nous avons essayé de décrire. Ayant focalisé notre analyse sur les stratégies exploitant l'argument de communauté dans la critique de cinéma, nous avons pu remarquer que cette orientation sur la communication de par sa nature délibérative pousse l'émetteur à réduire la distance temporelle et spatiale de son discours, ce qui est également cru pertinent dans le cas de la publicité.

L'adhésion du public s'appuie sur un schéma d'éclairage (Grize, 2004) susceptible de masquer toute autre perspective d'interprétation selon le principe de capter pour informer et informer pour capter. De ce fait, on obtient un movere efficace renforcé par un delectare stimulant : d'abord, on maintient l'identification aux valeurs prônées par l'émetteur (opération de généralisation) pour ensuite en augmenter l'impact (opération d'intensification) ( $c f$. Charaudeau, 2008). Par conséquent, la dimension rhétorique des stratégies employées relève de la planification d'effets liée à la persuasion qui prend en compte le sens, les savoirs et les intentions des interlocuteurs, soit les aspects socio-discursifs de l'argumentation ( $c f$. Amossy, 2015). C'est ainsi que l'intention communicationnelle de l'émetteur se projette dans des discours imprégnés de métonymies permettant de modeler la perception au niveau illocutoire de faire croire ainsi qu'au niveau perlocutoire de faire ressentir afin de focaliser notre attention sur des aspects concrets de la réalité décrite. Pour produire cet effet, le journaliste cherche à établir un lien interlocutif propice à la persuasion (relation de fiabilité) par la mise en relief des valeurs et des croyances supposées partagées qui doivent faciliter l'insertion du public dans l'univers du discours. La 
dimension persuasive et interprétative du genre de la critique imposerait donc une scénographie consistant à impliquer le récepteur dans le jeu interprétatif suggéré par l'émetteur, ce qui conduit le public à se persuader lui-même ( $c f$. aussi Grize, 2004). C'est pourquoi il serait intéressant d'examiner la question de savoir à quel point les stratégies persuasives conduisent à modeler l'interprétation du discours en s'adaptant aux goûts du public. Aussi serait-il possible de découvrir une panoplie de paramètres régissant l'attractivité du message, y compris son caractère ludique, ce qui permettrait, à notre sens, d'expliquer le fonctionnement de sous-genres nouveaux tels que des micro-critiques (cf. Wolny-Zmorzyński et al., 2014) où - comme dans le discours publicitaire - on cherche à consolider l'adhésion du public pour en éviter le regard critique sur les intentions et le contenu du dire. 


\section{BIBLIOGRAPHIE}

Amossy, R. (2008). Argumentation et Analyse du discours : perspectives théoriques et découpage disciplinaires. Argumentation et Analyse du Discours, 1. DOI: 10.4000/aad.200

Amossy, R. (2012). L'argumentation dans le discours. Nouvelle présentation ( $3^{\mathrm{e}}$ éd.). Paris : Armand Colin.

Amossy, R. (2015). Quelle vocation empirique pour l'argumentation dans le discours? Argumentation et Analyse du Discours, 15. DOI.org/10.4000/aad.2059

Baud, D. (2003). Analyse de genre : la critique de cinéma dans la presse quotidienne britannique. Asp, 39-40, pp. 37-45. DOI: $10.4000 /$ asp. 1282

Bonhomme, M., \& Pahud, S. (2013). Un renouveau actuel de la rhétorique publicitaire? Semen. Revue de sémio-linguistique des textes et discours, 36. Retrieved from http://semen.revues.org/9633

Breton, P. (2008). Convaincre sans manipuler. Apprendre à argumenter. Paris : La Découverte.

Brin, C., Charron, J., \& Bonville, J. de (2004). Nature et transformation du journalisme : théorie et recherches empiriques. Québec: Presses Université Laval.

Charaudeau, P. (2005). Les médias et l'information. L'impossible transparence du discours. Bruxelles : De Boeck.

Charaudeau, P. (2008). L'argumentation dans une problématique d'influence. Argumentation et Analyse $d u$ Discours, 1. DOI: 10.4000/aad.193

Charaudeau, P., \& Maingueneau, D. (2002). Dictionnaire d'analyse du discours. Paris : Seuil.

Doury, M., \& Plantin, Ch. (2015). Une approche langagière et interactionnelle de l'argumentation. Argumentation et Analyse du Discours, 15. DOI: 10.4000/aad.2006

Graff, R., \& Winn, W. (2006). Presencing “communion” in Chaim Perelman's New Rhetoric. Philosophy and Rhetoric, 39 (1), pp. 45-71.

Grize, J.-B. (2004). Le point de vue de la logique naturelle: démontrer, prouver, argumenter. In M. Doury, S. Moirand (éds), L'Argumentation aujourd'hui. Positions théoriques en confrontation (pp. 33-44). Paris : Presses Sorbonne Nouvelle.

Jacobs, S. (2015). Les principes pragmatiques de communication dans l'argumentation. Argumentation et Analyse du Discours, 15. DOI: 10.4000/aad.2078

Kerbrat-Orecchioni, C. (1980). L'énonciation de la subjectivité dans la langue. Paris : Armand Colin.

Kerbrat-Orecchioni, C. (2016). Axiologisation des termes affectifs et affectivisation des termes axiologiques : le cas des débats présidentiels. In A. Krzyżanowska \& K. Wołowska (éds), Les émotions et les valeurs dans la communication II. Entrer dans l'univers du discours (pp. 17-38). Frankfurt am Main : Peter Lang Edition.

Kövecses, Z. (2011). Język, umyst, kultura. Praktyczne wprowadzenie, trad. A. Kowalcze-Pawlik, M. Buchta. Kraków : Universitas [Oeuvre originale publiée 2006].

Lugrin, G. (2006). Généricité et intertextualité dans le discours publicitaire de presse écrite. Bern : Peter Lang.

Maingueneau, D. (2007). Genres de discours et modes de généricité. Le Français aujourd'hui, 4 (27). DOI: $10.3917 /$ lfa. 159.0029

Micheli, R. (2012). Les visées de l'argumentation et leurs corrélats langagiers : une approche discursive. Argumentation et Analyse du Discours, 9. DOI: 10.4000/aad.1406

Perelman, Ch. (2000). L'empire rhétorique. Rhétorique et argumentation. Paris : J. Vrin.

Petty, R. E., \& Cacioppo, J. T. (1986). Communication and persuasion: central and peripheral routes to attitude change (Springer series in social psychology). New York : Springer-Verlag.

Pietrzak, M. (2014). Recenzja i felieton na tle przemian prasy polskiej. Kilka uwag z ewolucji gatunku. In I. Jaros et al. (éds), Rozprawy Komisji Językowej ŁTN, t. LX (pp. 251-262). Łódź : Łódzkie Towarzystwo Naukowe. 
Plantin, Ch. (2011). Les bonnes raisons des émotions. Principes et méthode pour l'étude du discours émotionné. Berne : Peter Lang.

Rieffel, R. (2006). L'évolution du positionnement intellectuel de la critique culturelle. Quaderni, 60, pp. 55-64. DOI: 10.3406/quad.2006.2059

Robrieux, J. J. (2000). Rhétorique et argumentation. Paris : Nathan.

Stöckl, H. (2015). Mediolingwistyka. O statusie i metodach (nadal) konstytuującej się dziedziny badawczej, trad. A. Błaszczak. tekst $i$ dyskurs - text und diskurs, 8, pp. 37-61 [Oeuvre originale publiée 2012].

Wolny-Zmorzyński, K., Kaliszewski, A., Snopek, J., \& Furman, W. (2014). Prasowe gatunki dziennikarskie. Warszawa : Wydawnictwo Poltex. 